\title{
Empiema Pleural: Etiología, tratamiento y complicaciones*
}

\author{
Drs. JORGE SALGUERO A., GONZALO CARDEMIL H., JUAN CARLOS MOLINA F. ${ }^{\text {, }}$ \\ HANNS LEMBACH J. ${ }^{1}$, JAIME FERNÁNDEZ R. ${ }^{1,2}$ \\ Departamento de Cirugía, Hospital Clínico Universidad de Chile. \\ 2 Laboratorio de Comunicaciones Celulares, CEMC FONDAP, Facultad de Medicina, Universidad de Chile. \\ Santiago, Chile.
}

\begin{abstract}
Therapy of empyema. Retrospective review of 77 patients

Background: Empyema can cause significant medical problems, despite the advances in antimicrobial and surgical treatments. Aim: To compare the results of different therapeutic approaches to empyema. Material and Methods: Retrospective review of medical records of 77 patients with empyema (aged $53 \pm 20$ years, 48 males) treated in a clinical hospital between 1994 and 2007. Results: In 74\% of patients the cause of empyema was pneumonia. Thirty two were initially managed with percutaneous pleurostomy. Seven of these required and additional intervention due to residual empyema. Eleven patients were managed with open thoracotomy and 34 with video assisted thoracoscopy. Complications for percutaneous pleurostomy, open thoracotomy and video assisted thoracoscopy were 34,36 and $12 \%$ respectively. The figures for mortality were 25,0 and $0 \%$ respectively. Patients subjected to video assisted thoracoscopy required less days with a pleural tube than those subjected to percutaneous pleurostomy or open thoracotomy $(5,5-14,3$ and 12,4 days respectively). Conclusions: Video assisted thoracoscopy had the best therapeutic results for empyema in this series of patients.
\end{abstract}

Key words: Empyema, thoracoscopy, thoracotomy.

\section{Resumen}

Introducción: Definido como la presencia de infección bacteriana en el espacio pleural, determina una importante morbimortalidad a pesar de los avances en el tratamiento antibiótico y las nuevas técnicas quirúrgicas. Objetivos: Comparar resultados de las distintas estrategias terapéuticas para el Empiema Pleural (EP). Material y Métodos: Revisión retrospectiva de fichas clínicas de pacientes con diagnóstico de EP manejados en el Hospital Clínico Universidad de Chile entre enero de 1994 y junio de 2007. Se consideró significación estadística con $\mathrm{p}<0,05$. Resultados: De 77 pacientes, 48 fueron hombres y 29 mujeres, con edad promedio de 52,6 \pm 19,9 años. La causa más frecuente de EP fue la neumonía (74\%). En 55 pacientes se realizaron exámenes imagenológicos complementarios a la radiografía de tórax. Treinta y dos pacientes se manejaron inicialmente con pleurostomía percutánea (PP). Siete de ellos requirieron una nueva interven-

\footnotetext{
*Recibido el 16 de Junio de 2008 y aceptado para publicación el 29 de Enero de 2009.
}

Correspondencia: Dr. Jorge Salguero A.

Santos Dumont 999, Santiago, Chile

E-mail: jorsal@mi.cl 
ción por empiema residual. Once se manejaron con toracotomía abierta (TA) y 34 con Toracoscopia Vídeo asistida (TVA). La tasa de complicaciones y mortalidad fue: $34,37 \%$ y $25 \%$ para PP, $11,7 \%$ y $0 \%$ para TVA y $36,36 \%$ y $0 \%$ para TA, respectivamente (diferencias significativas). El tiempo de hospitalización promedio fue de 22,8 días, y el de tubo pleural de 9,21 días. Se encontró diferencias significativas entre los días con tubo pleural de los pacientes sometidos a TVA $(X=5,56)$ respecto de los sometidos a PP $(X=14,24)$ y TA $(\mathrm{X}=12,4)$. No se observan diferencias en tiempo de hospitalización. Conclusiones: La TVA demuestra obtener mejores resultados que la PP y la TA al evaluar mortalidad, complicaciones y días de tubo pleural.

Palabras clave: Empiema Pleural, toracoscopia vídeo asistida.

\section{Introducción}

El empiema pleural (EP), definido como la presencia de infección bacteriana en el espacio pleural, fue descrito por Hipócrates hace más de 2.400 años, quien ya en esa época practicaba el drenaje del espacio pleural mediante una incisión intercostal ${ }^{1-3}$. A pesar de los importantes avances en el campo de los antibióticos y las nuevas técnicas quirúrgicas, actualmente esta patología aún se asocia a una importante morbimortalidad ${ }^{4-6}$.

Se han planteado múltiples alternativas terapéuticas para el manejo de los EP; todas coinciden en el uso de antibióticos empíricos con cobertura de amplio espectro, agregando alguna técnica que logre el drenaje de la cavidad. Las distintas opciones incluyen la toracocentesis (única o múltiples), instalación de un tubo pleural (pleurostomía percutánea), la instilación de fibrinolíticos intrapleurales, y el drenaje a través de toracotomía abierta o toracoscopia vídeo asistida. No existe consenso respecto de cuál es la mejor alternativa para realizar el drenaje, ni el momento más propicio, pero sí se ha establecido que de un tratamiento rápido y eficiente depende el éxito de la evolución de esta patología $^{6-8}$. Estudios recientes sitúan a la TVA como una técnica mínimamente invasiva, con excelentes resultados en EP ya establecidos, en especial en aquellos refractarios a otras técnicas de drenaje. Dentro de sus ventajas destacan menor tasa de complicaciones, menor dolor post operatorio, rehabilitación más rápida y menor tiempo de hospitalización ${ }^{1,4}$.

En el presente trabajo se exponen 13 años de experiencia (1994-2007) en el manejo de pacientes con EP en Hospital Clínico de la Universidad de Chile, con especial énfasis en los resultados obtenidos a través de las distintas estrategias terapéuticas utilizadas.

\section{Material y Método}

Se realizó un estudio retrospectivo que consistió en la revisión de las fichas clínicas de los pacientes con diagnóstico de EP manejados en nues- tro centro entre los años 1994 y 2007. El criterio de inclusión fue el hallazgo de bacterias en el líquido pleural o $\mathrm{pH}$ bajo 7,15.

Se consignó edad, sexo y antecedentes mórbidos del paciente. Con respecto a la enfermedad actual se registró patología desencadenante, tiempo de hospitalización, post-procedimiento y retiro de tubos pleurales, e información referida a exámenes imagenológicos (radiografía de tórax, ecotomografía (ECO) y tomografía axial computarizada (TAC)) y a resultados del estudio del líquido pleural obtenido a través de la toracocentesis diagnóstica $(\mathrm{pH}, \mathrm{LDH}$, Glucosa, Gram y cultivo). Se evaluaron las distintas estrategias terapéuticas considerando el tiempo de permanencia del drenaje pleural, el tiempo de estadía hospitalaria, las complicaciones y la mortalidad.

El análisis estadístico se realizó utilizando la prueba de t-Student para la comparación de variables continuas y chi-cuadrado o Fisher para la comparación de proporciones. Se consideró como significativo un valor de $\mathrm{p}<0,05$.

\section{Resultados}

La serie quedó compuesta por 77 pacientes, 48 hombres $(62,34 \%$ ) y 29 mujeres $(37,66 \%)$, con una edad promedio al momento del diagnóstico de $52,66 \pm 19,9$ y un rango entre 10 y 93 años (Tabla 1).

Cincuenta y cinco pacientes $(71,4 \%)$ presentaban por lo menos una comorbilidad, la mayor parte de ellos patología cardiovascular (21 pacientes), seguido de diabetes mellitus (15 pacientes), y daño hepático crónico ( 9 pacientes). La ubicación más frecuente fue derecha, con 40 pacientes, 36 izquierdos y 1 bilateral. La etiología del empiema fue en la mayor parte de los casos derrame paraneumónico (57 pacientes, $74,03 \%$ de los casos).

Todos los pacientes de nuestra serie fueron estudiados inicialmente con una radiografía de tórax que evidenció el derrame. Cincuenta y cuatro pacientes, correspondientes al 70,12\% fueron estudiados con algún método imagenológico complementario: 16 sólo TAC, 17 sólo ECO y 21 ambos. Dentro de los hallazgos imagenológicos, 49 (63,3\%) 
Tabla 1. Características generales de la serie

\begin{tabular}{lcc}
\hline Serie & n & \% \\
\hline Total pacientes & 77 & 100,00 \\
Hombres & 48 & 62,34 \\
Mujeres & 29 & 37,66 \\
Edad al diagnóstico (X/DS) & $52,66 \pm 19,9$ \\
& & \\
Ubicación & & \\
$\quad$ Izquierdo & 36 & 46,75 \\
$\quad$ Derecho & 40 & 51,95 \\
$\quad$ Bilateral & 1 & 1,30 \\
& & \\
Comorbilidad & $\square$ & $\square$ \\
$\quad$ Cardiovascular & 21 & 37,50 \\
$\quad$ Diabetes & 15 & 26,79 \\
DHC & 9 & 16,07 \\
Patología respiratoria & 7 & 12,50 \\
IRC & 4 & 7,14 \\
Total pacientes con comorbilidad & 55 & 71,43 \\
Patología Primaria & & \\
Neumonía & & \\
Trauma & 57 & 74,03 \\
Post quirúrgico & 2 & 2,60 \\
Otros & 3 & 3,90 \\
\hline
\end{tabular}

DHC: Daño hepático crónico.

IRC: Insuficiencia renal crónica.

Tabla 2. Hallazgos del estudio citoquímico del líquido pleural

\begin{tabular}{lrrr}
\hline & \multicolumn{1}{c}{ X } & \multicolumn{1}{c}{ DS } & \multicolumn{1}{c}{ Rango } \\
\hline $\mathrm{pH}$ & 6,71 & 0,29 & $6,18-7,17$ \\
Glucosa & 39,32 & 46,45 & $<20-209$ \\
LDH & $9.036,17$ & $15.050,85$ & $215-68.900$ \\
\hline
\end{tabular}

correspondieron a EP tabicados, y 28 (36,4\%) fueron diagnosticados como EP libres.

En el análisis citoquímico del líquido extraído a través de toracocentesis destaca que todos los pacientes presentaban un $\mathrm{pH}$ menor de 7,17 , con un promedio $6,71 \pm 0,29$, y un valor promedio de glucosa de 39,32 \pm 46,45 (Tabla 2). Se evidenciaron microorganismos a través de tinción de Gram sólo en el $25,97 \%$ (20 pacientes), principalmente cocaceas Gram $(+)$ en el $60 \%$ de éstos. El cultivo del líquido pleural fue positivo en un $25,97 \%$ (20 pacientes), siendo los gérmenes más frecuentemente aislados las cocaceas Gram $(+)$ en el $50 \%$ y bacilos Gram (-) en el 40\% de las muestras. No se desarrollaron anaerobios en los cultivos realizados.

Treinta y dos pacientes $(41,55 \%)$ fueron manejados inicialmente a través de pleurostomía, de los cuales 16 evolucionaron satisfactoriamente, 2 presentaron complicaciones que se manejaron de manera conservadora ( 1 shock séptico y 1 fístula bronco-pleural), 7 debieron ser sometidos a otro procedimiento por mala evolución clínica (5 TVA y 2 TA, 1 de los cuales falleció posteriormente) y 8 fallecieron: 7 de manera precoz y 1 luego de una TA (Tabla 3). En 11 pacientes (14,28\%) el manejo inicial fue a través de una TA, de los cuales 7 evolucionaron satisfactoriamente, y 4 evolucionaron con complicaciones requiriendo uno de ellos reintervención a causa de una sepsis de foco pleural. Los restantes 34 pacientes $(44,15 \%)$ fueron manejados inicialmente con TVA, presentando una tasa de complicaciones post-operatorias de 8,82\% (3 pacientes: 2 sepsis severas y una neumonía nosocomial que recibieron tratamiento médico). Ninguno de los pacientes sometidos a esta intervención requirió un nuevo procedimiento ni falleció.

De los 28 pacientes con EP catalogados por imágenes como libres, 23 se trataron inicialmente con PP (Figura 1), de los cuales 7 fallecieron; 1 presentó un shock séptico que se manejó de manera conservadora y 1 requirió una TVA por mala evolución. Cinco se manejaron inicialmente con TVA, uno

Tabla 3. Complicaciones y mortalidad según tratamiento inicial

\begin{tabular}{|c|c|c|c|c|c|c|}
\hline & \multicolumn{2}{|c|}{ Complicaciones } & \multicolumn{2}{|c|}{ Mortalidad } & \multicolumn{2}{|c|}{ Total pacientes } \\
\hline & $\mathbf{n}$ & $\%$ & $\mathbf{n}$ & $\%$ & $\mathbf{n}$ & $\%$ \\
\hline Pleurostomía percutánea & 16 & $(50)^{*}$ & 8 & $(25)^{*}$ & 32 & $(41,56)$ \\
\hline TVA & 3 & $(8,82)^{*}$ & $0 *$ & & 34 & $(44,16)$ \\
\hline Toracotomía abierta & 4 & $(36,36)^{*}$ & $0 *$ & & 11 & $(14,28)$ \\
\hline Total & 23 & $(29,87)$ & 8 & $(10,39)$ & 77 & $(100)$ \\
\hline
\end{tabular}

\footnotetext{
* Diferencia estadísticamente significativa, $\mathrm{p}<0,05$.
} 
Tabla 4. Tiempo de hospitalización, estadía post-procedimiento y días de tubo pleural según tratamiento

\begin{tabular}{lcccccc} 
& \multicolumn{2}{c}{ Días hospitalización } & Días post procedimiento & \multicolumn{2}{c}{ Días tubo pleural } \\
& $\mathbf{X}$ & DS & $\mathbf{X}$ & DS & X & DS \\
\hline Pleurostomía percutánea & 26,25 & 21,36 & 15,12 & 13,34 & $14,24^{*}$ & 13,06 \\
TVA & 20,82 & 15,10 & 9,78 & 11,74 & $5,56^{*}$ & 3,04 \\
Toracotomía & 22,82 & 11,46 & 14,40 & 10,16 & $12,40^{*}$ & 9,09 \\
Pleurostomía + TVA & 17,60 & 7,92 & 7,80 & 2,28 & 5,40 & 4,51 \\
Pleurostomía + toracotomía & 25,50 & 17,68 & 5,00 & 1,41 & 9,50 & 3,54 \\
Promedio & 22,87 & 16,45 & 11,70 & 11,49 & 9,21 & 8,92 \\
\hline
\end{tabular}

*Diferencia estadísticamente significativa, $\mathrm{p}$ Value $<0,05$.

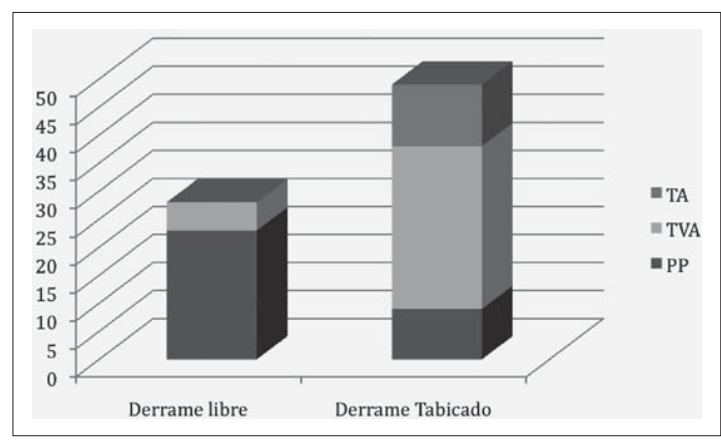

Figura 1. Tratamiento inicial del EP según hallazgos imagenológicos. TA: Toracotomía abierta; TVA: Toracoscopia vídeo asistida; PP: Pleurostomía percutánea.

de los cuales evolucionó con cuadro séptico que fue manejado sin requerir nuevos procedimientos. De los 49 con EP catalogados como tabicados 9 fueron manejados inicialmente con PP, de los cuales 2 evolucionaron favorablemente y 7 presentaron complicaciones por las cuales 6 requirieron otra intervención: cuatro se manejaron con TVA y 2 con TA. Once pacientes fueron manejados inicialmente con TA, 4 de los cuales presentaron complicaciones ( 1 requirió una nueva TA, los otros 3 se manejaron de manera conservadora), sin presentarse mortalidad. Los restantes 29 pacientes con EP tabicados fueron manejados inicialmente con TVA, 3 de los cuales presentaron complicaciones que se manejaron de manera conservadora. Ninguno de los pacientes sometidos inicialmente a TVA requirió un nuevo procedimiento ni falleció.

La mortalidad general de la serie fue de 10,39\% (8 pacientes), todos ellos pertenecientes al grupo que inicialmente se trató con pleurostomía. De estos, 3 fallecieron por falla multiorgánica secundaria al proceso séptico y 5 por shock séptico. La tasa de complicaciones fue de $29,87 \%$ (23 pacientes, la mayor parte de ellos sepsis severa o shock séptico).
Si se excluye a los pacientes que requirieron más de un procedimiento, la comparación de la tasa de complicaciones entre los pacientes sometidos a TVA $(8,82 \%)$ con respecto a los sometidos a PP $(36 \%)$ y a TA $(30 \%)$, muestra diferencias estadísticamente significativas $(p=0,009)$. La comparación entre las tasas de mortalidad según las mismas condiciones, también demuestra ser estadísticamente significativa $(0 \%$ para TVA, $0 \%$ para TA y $28 \%$ para $\mathrm{PP})$ con $\mathrm{p}=0,004$ (Tabla 3).

Esta tendencia se mantiene si se considera como complicación la necesidad de una nueva intervención y se incluyen por lo tanto, quienes recibieron un segundo tratamiento. En este caso las tasas de complicación son de $8,82 \%$ para TVA, $50 \%$ para PP y $36,36 \%$ para TA. Esta diferencia también es estadísticamente significativa, con un $\mathrm{p}=0,003$ (Tabla 3 ).

El tiempo de hospitalización promedio de la serie fue de 22,87 $\pm 16,45$ días, el tiempo de hospitalización post-procedimiento de 11,7 $\pm 11,49$ y el de tubo pleural de 9,21 $\pm 8,92$ (Tabla 4). No se encontraron diferencias significativas entre los grupos de pacientes manejados con TVA, PP y TA en relación al tiempo de hospitalización ni el tiempo de estadía hospitalaria post-procedimiento, pero sí al comparar los días de tubo pleural entre los pacientes sometidos a TVA $(\mathrm{X}=5,56)$ con respecto a los sometidos a PP $(14,24)$ y TA $(12,40)(\mathrm{p}<0,002)$.

\section{Discusión}

El proceso fisiopatológico que lleva a la formación de un EP corresponde a un continuo que comienza con un exudado simple, generalmente un derrame pleural paraneumónico, que de no mediar tratamiento evoluciona a una etapa fibrinopurulenta con infección del líquido pleural por bacterias y formación de pus, para finalmente llegar a la orga- 
nización y tabicación del derrame. Es muy importante considerar que el diagnóstico de EP se basa en la presencia de pus, y no en el análisis de laboratorio del líquido pleural ${ }^{9,10}$.

Considerando esta continuidad fisiopatológica entre el derrame pleural y el desarrollo de empiema, el American College of Chest Physicians desarrolló una nueva clasificación para estas dos entidades publicada en el 2000 en la cual se separan 4 grupos según el riesgo de presentar una evolución desfavorable de la enfermedad, considerando 3 variables: los cambios imagenológicos del espacio pleural, la bacteriología y el análisis bioquímico del líquido pleural. Así, la indicación de drenaje está reservada para los pacientes con riesgo moderado y alto, es decir, pacientes con derrames masivos, mayores al $50 \%$ del hemitórax, engrosamiento pleural, tabicamiento, presencia de bacterias en el líquido pleural (evidenciadas por tinción de gram o cultivos) o $\mathrm{pH}$ $\leq 7,2^{11}$. A pesar de los avances diagnósticos y terapéuticos el empiema pleural sigue siendo una complicación grave asociada a mortalidad en múltiples series. En pacientes con neumonía adquirida en la comunidad, la aparición de un derrame pleural (y su posible complicación) ocurre en 20 a $40 \%$ de los hospitalizados, y este hecho eleva su mortalidad entre 3 y 6 veces dependiendo si el derrame es uni o bilateral ${ }^{9}$. Salvo excepciones requiere drenaje quirúrgico, sin embargo, la técnica más apropiada y el momento quirúrgico son interrogantes aún no respondidas categóricamente por la evidencia disponible.

Los 77 pacientes con diagnóstico de EP estudiados en nuestro centro muestran un perfil epidemiológico similar a otras publicaciones internacionales, predominando los pacientes de sexo masculino, portadores de enfermedades crónicas en su mayoría, con una edad promedio en torno a los 50 años de edad ${ }^{12}$. Al igual que publicaciones extranjeras y nacionales, nuestra serie muestra a la complicación de una neumonía adquirida en la comunidad como la principal causa de esta patología $a^{13-16}$.

Respecto a los microorganismos más frecuentemente involucrados, la literatura clásica ha demostrado un predominio de gérmenes gram $(+)$, seguido de Gram (-) y con participación menor de gérmenes anaerobios. La tendencia en las series publicadas es al aumento de los Gram (-) y anaerobios ${ }^{17,18}$, mostrando la literatura nacional ${ }^{13-15}$ una tendencia similar. En nuestra serie los gérmenes aislados correspondieron principalmente a Cocaceas Gram $(+)$ y Bacilos Gram (-), sin constituir los anaerobios una etiología importante.

La ECO y TAC como imágenes complementarias a la radiografía de tórax, son las herramientas diagnósticas de elección en derrames complicados de evolución tórpida. Los hallazgos incluyen mal posición del drenaje pleural, engrosamiento pleural y frecuentemente tabicación del empiema ${ }^{9}$. En nuestra serie, $70 \%$ de los pacientes requirió estudio complementario, encontrándose tabicación en un $57 \%$ de ellos (49 pacientes). Estos hallazgos permitieron la orientación del equipo tratante hacia técnicas de drenaje pleural más invasivas, siendo la TVA la más utilizada (59\% de los EP tabicados), mientras en los EP libres se prefirió la instalación de PP como manejo inicial (82\% de los EP libres).

La literatura muestra que el éxito de cada técnica de drenaje pleural depende del momento en la evolución del derrame en que se aplique. Así técnicas menos invasivas como la pleurocentesis o PP aplicadas en derrames en fase exudativa o fibrinopurulenta inicial, pueden tener resultados satisfactorios. Sin embargo, ante empiemas establecidos y en especial en aquellos en fase organizada, el rendimiento de tales técnicas se reduce significativamente, aumentando la tasa de complicaciones y mortalidad, y necesitando someter a estos pacientes a procedimientos progresivamente más invasivos para lograr resolver el cuadro. Se ha reportado entre un 36 y $65 \%$ de fracaso en el drenaje mediante PP y antibióticos en empiemas iniciales ${ }^{12}$. Se ha reportado una disminución de esta tasa de fracaso cuando la instalación del tubo pleural se realiza guiada por imágenes. Una serie de 103 pacientes con EP drenados mediante tubo pleural guiado por TAC o Ecografía muestra un mayor rendimiento (78\% éxito) como método definitivo de tratamiento $^{19}$.

En nuestra serie, 32 pacientes $(41,55 \%)$ se manejaron inicialmente con PP. Veintitrés de ellos tenían EP libres a la TAC o ECO, y de ellos 14 (60\%) presentaron una evolución satisfactoria. Por otro lado, de los 9 pacientes con EP tabicados manejados inicialmente con PP, sólo 2 tuvieron evolución satisfactoria (22\%), 1 evolucionó con complicaciones que se manejaron de manera conservadora y 6 necesitaron TVA o TA, falleciendo uno de ellos por complicaciones sépticas. Este análisis nos demuestra que, en nuestra serie, la PP como tratamiento del EP tiene mejor rendimiento en fases iniciales, y en los casos más avanzados el rendimiento es pobre necesitando frecuentemente otros procedimientos más invasivos y concentrando buena parte de las complicaciones y mortalidad de la serie.

La literatura muestra también que cuando se detecta a los pacientes con EP en etapas avanzadas y se realizan procedimientos más invasivos de entrada (TVA o TA), se logra mayor tasa de éxito, menor tasa de complicaciones y hospitalizaciones más breves ${ }^{9,10,20}$. Respecto a la TVA en particular, se menciona en la literatura como un procedimiento 
intermedio en términos de invasividad ${ }^{9}$, con excelentes resultados en empiemas refractarios a otros procedimientos. Un análisis de 9 trabajos de TVA en EP, que agrupa 580 pacientes, muestra una tasa de éxito en torno al $83 \%{ }^{10}$, demostrando ser un procedimiento superior a la toracocentesis a repetición, PP y uso de fibrinolíticos intrapleurales ${ }^{20}$. Siendo de probada utilidad en el manejo de EP tabicados, la limitante actual del uso de la TVA está en los pacientes con EP altamente organizados formando membranas o "peel" pleurales, cuya decorticación requiere a menudo conversión a TA. En nuestra serie 34 pacientes $(44 \%)$ se manejaron con TVA inicialmente, con una tasa general de éxito sin complicaciones del 91\%. Tres pacientes $(8,8 \%)$ evolucionaron con complicaciones, todas ellas sépticas, siendo esta tasa significativamente menor que los pacientes manejados con PP y TA. Ninguno de los pacientes manejados con TVA inicialmente requirió otros procedimientos adicionales, y no hubo mortalidad en este grupo. Por otro lado, hubo 5 pacientes sometidos a TVA posterior a una PP fallida, con evolución sin complicaciones en 4 de ellos $(80 \%)$, y sin registrar mortalidad. El análisis de estos resultados muestra que en nuestra serie, la TVA fue un procedimiento altamente exitoso, que resolvió la patología en la totalidad de los pacientes tratados, presentando la tasa más baja de complicaciones, y con claros beneficios en cuanto a estadía hospitalaria y sobrevida de los pacientes.

\section{Conclusión}

Los resultados de nuestra serie ubican a la TVA como el procedimiento de primera elección para el tratamiento del EP, en especial para aquellos tabicados o refractarios a otros medios de drenaje. Dentro de sus ventajas destacan menor tasa de complicaciones, de mortalidad, rehabilitación más rápida y menor tiempo de hospitalización.

\section{Referencias}

1. Magovern C, Rusch V. Parapneumonic and posttraumatic pleural space infections. Chest Surg Clin N Am 1994; 4: 561-582.

2. Chadwick J, Mann WN. Medical works of Hippocrates. Oxford: Blackwell, 1950: 121.

3. Allamand J. Empiema Pleural no Tuberculoso, Afec- ción Médico Quirúrgica, evolución de su conocimiento. Enferm Respir Cir Torax 1989; 5: 150-165.

4. Ferguson A, Prescott R, Selkon J, Watson D, Swinburn C. The clinical course and management of thoracic empyema. Quart J Med 1996; 89: 285-289.

5. Sahn S. Management of complicated parapneumonic effusion. Am Rev Respir Dis 1993; 148: 813-817.

6. Mwandumba H, Beeching N. Pyogenic lung infections: factors for predicting clinical outcome of lung abscess and thoracic empyema. Curr Opin Pulm Med 2000; 6: 234-239.

7. Huang H, Chang H, Chen C, Lee C, Hsiue T. Predicting Factors for Outcome of Tube Thoracostomy in Complicated Parapneumonic Effusion or Empyema. CHEST 1999; 115: 751-756.

8. Laisaar T, Ilves A. Surgical Treatment of Pleural Empyema. Scand Cardiovasc J 1999; 33: 97-102.

9. Light R. Parapneumonic effusions and empyema. Proc Am Thorac Soc 2006; 3: 75-80.

10. Schiza S, Siafakas N. Clinical presentation and management of empyema, lung abscess and pleural effusion. Curr Opin Pulm Med 2006; 12: 205-211.

11. Colice G, Curtis A, Deslauriers J, Heffner J, Light R, Littenberg B, et al. Medical and surgical treatment of parapneumonic effusions: an evidence-based guideline. Chest 2000; 118: 1158-1171.

12. Luh S, Shou M, Wang L, Chen J, Tsai T. Video assisted toracoscopic surgery in the treatment of complicated parapneumonic effusions or empyemas. Chest 2005; 127: 1427-1432.

13. Cornejo C. Compilado Carlos Cornejo. Rev Chil Cir 1988; 40: 67.

14. Álvarez R, Cacciuttolo G, Rocabado J. Empiema pleural. Cuad Cir 1996; 10: 53-58.

15. Benavides F, Valeria C, Medina B. Empiema Pleural. Bol Hosp SJ de Dios 1996; 43: 187-192.

16. Heffner J. Infection of the pleural space. Clin Chest Med 1999; 20: 607-622.

17. Cardemil G. Empiema pleural. En: Castro J, Hernández G, ed. Sepsis. Santiago: Editorial Mediterráneo, 1993.

18. Chen K, Hsueh P, Liaw, Yang P, Luh K. A 10-Year Experience with Bacteriology of Acute Thoracic Empyema Chest 2000; 117: 1685-1689.

19. Shankar S, Gulati M, Kang M, Gupta S, Suri S. Imageguided percutaneous drainage of thoracic empyema: can sonography predict the outcome? Eur Radiol 2000; 10: 495-499.

20. Coote N, Kay E. Surgical versus non-surgical management of pleural empyema. Cochrane Database Syst Rev 2005; 4. Disponible en: www.cochrane.org (Consultado el 18 de octubre de 2007). 